AIP /123-QED

\title{
Determination of the complex refractive index and optical bandgap of $\mathrm{CH}_{3} \mathrm{NH}_{3} \mathrm{Pbl}_{3}$
} thin films

\author{
J. A. Guerra, ${ }^{1,2, a)}$ A. Tejada $^{2}{ }^{2}$ L. Korte, ${ }^{1, b)}$ L. Kegelmann, $^{1}$ J. A. Töfflinger, ${ }^{2} \mathrm{~S}$. \\ Albrecht, ${ }^{3}$ B. Rech, ${ }^{1}$ and R. Weingärtner ${ }^{2}$ \\ 1) Helmholtz-Zentrum Berlin für Materialien und Energie GmbH, \\ Institut für Silizium-Photovoltaik, Kekuléstraße 5, 12489 Berlin, \\ Germany. \\ ${ }^{2)}$ Departamento de Ciencias, Sección Fúsica, Pontificia \\ Universidad Católica del Perú, Av. Universitaria 1801, Lima 32, \\ Peru. \\ 3) Helmholtz-Zentrum Berlin für Materialien und Energie GmbH, \\ Young Investigator Group for Perovskite Tandem Solar Cells, Kekuléstraße 5, \\ 12489 Berlin, Germany.
}

(Dated: 22 November 2017)

We report the complex refractive index of methylammonium lead iodide $\left(\mathrm{CH}_{3} \mathrm{NH}_{3} \mathrm{PbI}_{3}\right)$ perovskite thin films obtained by means of variable angle spectroscopic ellipsometry and transmittance/reflectance spectrophotometry in the wavelength range of $190 \mathrm{~nm}$ to $2500 \mathrm{~nm}$. Film thickness and roughness layer thickness are determined by minimizing a global unbiased estimator in the region where the spectrophotometry and ellipsometry spectra overlap. We then determine the optical bandgap and Urbach energy from the absorption coefficient, by means of a fundamental absorption model based on band fluctuations in direct semiconductors. This model merges both the Urbach tail and the absorption edge regions in a single equation. In this way, we increase the fitting region and extend the conventional $(\alpha \hbar \omega)^{2}$-plot method to obtain accurate bandgap values.

\footnotetext{
a)Electronic mail: guerra.jorgea@pucp.edu.pe

b)Electronic mail: korte@helmholtz-berlin.de
} 

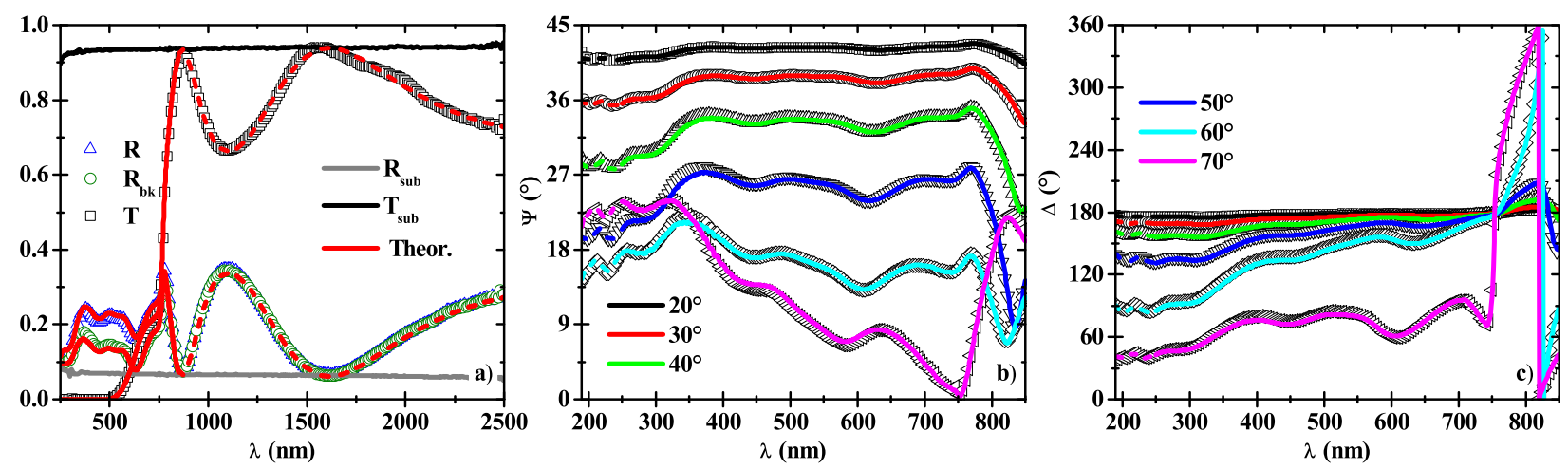

FIG. 1. Reflectance $(R)$, back-side reflectance $\left(R_{\mathrm{bk}}\right)$ and transmittance $(T)(\mathrm{a}), \Psi$ (b) and $\Delta(\mathrm{c})$ spectra. Solid lines correspond to theoretical curves in the overlapping region between the different techniques (250 nm to $850 \mathrm{~nm}$ ). Dashed lines correspond to the theoretical curves calculated from VASE only (below $250 \mathrm{~nm}$ ) and from spectrophotometry only (above $850 \mathrm{~nm}$ ). $R_{\text {sub }}$ and $T_{\text {sub }}$ stand for the substrate's reflectance and transmittance, respectively.

\section{INTRODUCTION}

Organic-inorganic hybrid perovskite materials have gained increasing attention in recent years $^{1-13}$. This is mainly attributed to their strong optical absorption, associated to a direct bandgap ${ }^{2}$, long carrier diffusion length ${ }^{3}$, and low-cost fabrication methods, ${ }^{4}$ which are particularly suitable features for photovoltaic applications. In this matter, perovskitebased solar cells have achieved a power conversion efficiency of up to $22.1 \%$, and this value is still far from the theoretical efficiency limit $^{5}$. They have also been proposed for the top cell in monolithic silicon-based tandem solar cells ${ }^{8,9}$. Finally, their bandgap can be tailored ${ }^{10,11}$, serving also as an appropriate antireflection coating due to a tunable refractive index ${ }^{6-8}$.

Accurate knowledge of the complex refractive index $(\tilde{n})$, optical bandgap $\left(E_{\mathrm{g}}\right)$ and $\operatorname{Urbach}$ energy $\left(E_{\mathrm{u}}\right)$ is essential for the design of optoelectronic devices. However, these quantities are usually obtained from optical data by fitting a dispersion model, which can introduce unwanted biases. Additional bias is introduced in the bandgap determination, when obtained from the fundamental absorption, due to a small and arbitrary fitting region. The complex refractive index of $\mathrm{CH}_{3} \mathrm{NH}_{3} \mathrm{PbI}_{3}$ perovskite thin films, the effect of air exposure and the absorption coefficient overestimation due to the surface roughness have been recently reported. A review can be found in the work of Shirayama et $\mathrm{al}^{1}$. 
In this work we report $\tilde{n}, E_{\mathrm{g}}$ and $E_{\mathrm{u}}$ of $\mathrm{CH}_{3} \mathrm{NH}_{3} \mathrm{PbI}_{3}$ perovskite thin films. $\tilde{n}$ is determined by means of variable angle spectroscopic ellipsometry (VASE) and transmittance/reflectance spectrophotometry in the wavelength range of $190 \mathrm{~nm}$ to $2500 \mathrm{~nm}$. We take advantage of both spectrophotometry and ellipsometry spectra to set up an overconstrained system of equations for $n$ and $k(\tilde{n} \equiv n-i k)$. This data set is composed by the transmittance $(T)$, reflectance $(R)$ and back-side reflectance $\left(R_{\mathrm{bk}}\right)$ at near normal incidence, along with ellipsometry $\Psi$ and $\Delta$ spectra taken at six different incident angles. Film thickness $\left(d_{\mathrm{f}}\right)$ and roughness layer thickness $\left(d_{\mathrm{r}}\right)$ are estimated by a global error minimization approach. We compare the obtained $\tilde{n}$ with the ones recently published by Shirayama et $\mathrm{al}^{1}$ and Löper et $\mathrm{al}^{7}$. In order to retrieve $E_{\mathrm{g}}$ and $E_{\mathrm{u}}$, we develop a simple and straightforward model based on band fluctuations in the Joint Density of States (JDOS) to describe the fundamental absorption of direct semiconductors. The model requires only three fitting parameters. An asymptotic analysis of the fundamental absorption model reveals the expected square root behaviour above the bandgap and the universal exponential tail below it.

\section{EXPERIMENTAL DETAILS}

The $\mathrm{CH}_{3} \mathrm{NH}_{3} \mathrm{PbI}_{3}$ perovskite layer was grown on a two side polished $500 \mu \mathrm{m}$ thick quartz substrate, according to the procedure described by Jeon et al ${ }^{12} \cdot 1.1 \mathrm{M} \mathrm{CH}_{3} \mathrm{NH}_{3} \mathrm{I}(\mathrm{MAI}$, Dyenamo) and 1.1 $\mathrm{M} \mathrm{PbI}_{2}(99.99 \%$, TCI) were first dissolved in a cosolvent of $\gamma$-butyrolactone (GBL, $\geq 99 \%$, Sigma Aldrich) and dimethyl sulfoxide (DMSO, Sigma-Aldrich) with a 7:3 volume ratio and left stirring at $60^{\circ} \mathrm{C}$ for 12 hours. The precursor solution was then spin coated on the samples in three consecutive steps at $1000 \mathrm{rpm}$ for 10 seconds, $2000 \mathrm{rpm}$ for 20 seconds and $5000 \mathrm{rpm}$ for 10 seconds, with $150 \mu \mathrm{l}$ of toluene poured on during the third spinning stage in order to improve the crystal growth uniformity. A subsequent annealing at $100^{\circ} \mathrm{C}$ for 10 minutes completed the perovskite layer preparation. This process is equivalent to the one used by Löper ${ }^{7}$. The layer is relevant to solar cells and currently achieves up to $18 \%$ PCE in devices.

The spectral $T, R$ and $R_{\mathrm{bk}}$ curves were measured with a Perkin Elmer Lambda 1050 spectrophotometer with an integrating sphere in the wavelength range of $250 \mathrm{~nm}$ to 2500

$\mathrm{nm}$ (5 nm step). The VASE measurements were performed with a Sentech 850 ellipsometer from $190 \mathrm{~nm}$ to $850 \mathrm{~nm}(\sim 1 \mathrm{~nm}$ step). 


\section{COMPLEX REFRACTIVE INDEX DETERMINATION}

There are some aspects to consider in the calculation of the optical constants. On the one hand, ellipsometry is very sensitive to the sample surface. It is therefore necessary to consider the surface roughness, even for a small roughness layer thickness ${ }^{1,14}$. On the other hand, spectrophotometry is quite robust to roughness ${ }^{15}$. Its presence can go unnoticed even with a $10 \mathrm{~nm}$ roughness layer. As a practical rule, the roughness effect is negligible if the maxima (minima) of the transmittance (reflectance) oscillations coincide with the bare substrate's transmittance (reflectance) in the transparent and low absorption regions, as depicted in Fig. 1. We use the Bruggeman effective medium approximation (EMA) with a void volume fraction $f_{\text {void }}=50 \%^{1,7,14}$ to model the roughness as an additional layer, of thickness $d_{\mathrm{r}}$, between the ambient and the perovskite thin film.

Usually, in order to retrieve $n$ and $k$ from an insufficiently constrained system of equations such as $\Psi$ and $\Delta$, or $R$ and $T$, for each wavelength, a model for the complex refractive index is chosen. In this way, the number of unknown variables is reduced from $2 \mathrm{~N}+2$, where $\mathrm{N}$ is the number of data points, to a manageable number of fitting parameters. If $d_{\mathrm{f}}$ and $d_{\mathrm{r}}$ are known, then $n$ and $k$ can be obtained by a mathematical inversion ${ }^{14}$ from two independent spectral curves. Nevertheless, the latter approach is usually very sensitive to the exact film structure that is assumed, as well as measurement noise. This can lead to very noisy results and/or nonphysical artifacts. In the dispersion modeling approach, the selection of a suitable model can be troublesome, especially when attempting to determine the optical bandgap and Urbach energy. Although in the case of metal halide perovskites, the Forouhi-Bloomer dispersion model seems to be very suitable, when looking beyond the fundamental absorption region, the addition of more than three oscillators is necessary ${ }^{1}$. This considerably increases the number of fitting parameters to determine, thus hindering the method if no suitable starting values are known ${ }^{14}$.

It is possible to introduce additional constraints by increasing the number of measured spectral curves ${ }^{7,15}$. For instance, this can be done by measuring spectral ellipsometry of a second sample with the same optical constants but different film thickness. $\tilde{n}$ is obtained from the first sample by performing a mathematical inversion and then used in a fitting procedure on the spectral data of the second sample to determine $d_{\mathrm{r}}$ and $d_{\mathrm{f}}{ }_{\mathrm{f}}^{1,14}$. However, since obtaining two samples with identical optical constants sometimes might also be a 
challenge, additional adjustments to the calculated layers thicknesses might be necessary in order to obtain positive values for the imaginary part of the dielectric constant below the bandgap ${ }^{1}$.

In this work, however, we increase the number of measured spectral curves from one sample by using $\Psi$ and $\Delta$ obtained at six different incident angles, as well as $R, R_{\mathrm{bk}}$ and $T$ obtained at normal incidence, thus achieving the necessary redundancy by using two separate measurement techniques on one sample, rather than two separate samples. For a given set of $d_{\mathrm{r}}$ and $d_{\mathrm{f}}, \tilde{n}\left(\lambda_{i}\right)$ can be calculated by minimizing the global unbiased estimator $\sigma_{i}^{2}$ at each wavelength $\lambda_{i}$, independently. The sub-index $i$ runs from 1 to $\mathrm{N}$, the number of data points.

$$
\sigma_{i}^{2}=\sigma_{\mathrm{VASE}}^{2}\left(n_{i}, k_{i}, d_{\mathrm{r}}, d_{\mathrm{f}}\right)+\sigma_{\mathrm{RT}}^{2}\left(n_{i}, k_{i}, d_{\mathrm{f}}\right),
$$

Here, $\sigma_{\mathrm{VASE}}^{2}$ and $\sigma_{\mathrm{RT}}^{2}$ are given in Eqs. 2 and 3, respectively.

$$
\begin{aligned}
& \sigma_{\mathrm{VASE}}^{2}\left(n_{i}, k_{i}, d_{\mathrm{r}}, d_{\mathrm{f}}\right)= \\
& \sum_{j=1}^{6}\left[\operatorname{Re}\left({ }^{\mathrm{th}} \rho\left(n_{i}, k_{i}, d_{\mathrm{r}}, d_{\mathrm{f}}, \theta_{j}\right)\right)-\operatorname{Re}\left({ }^{\mathrm{me}} \rho_{i j}\right)\right]^{2} \\
& +\left[\operatorname{Im}\left({ }^{\text {th }} \rho\left(n_{i}, k_{i}, d_{\mathrm{r}}, d_{\mathrm{f}}, \theta_{j}\right)\right)-\operatorname{Im}\left({ }^{\mathrm{me}} \rho_{i j}\right)\right]^{2} \\
& \sigma_{\mathrm{RT}}^{2}\left(n_{i}, k_{i}, d_{\mathrm{f}}\right)= \\
& \left({ }^{\text {th }} R_{\mathrm{bk}}\left(n_{i}, k_{i}, d_{\mathrm{f}}\right)-{ }^{\mathrm{me}} R_{\mathrm{bk} i}\right)^{2} \\
& +\left({ }^{\text {th }} R\left(n_{i}, k_{i}, d_{\mathrm{f}}\right)-{ }^{\text {me }} R_{i}\right)^{2} \\
& +\left({ }^{\text {th }} T\left(n_{i}, k_{i}, d_{\mathrm{f}}\right)-{ }^{\text {me }} T_{i}\right)^{2}
\end{aligned}
$$

The left upper-indexes "th" and "me" stand for theoretical and measured, respectively. $\rho$ is the ellipsometric variable defined as $\rho=\tan (\Psi) \exp (i \Delta) . \theta_{j}$ is the incident angle and the sub-index $j$ runs from 1 to the maximum number of used incident angles. The theoretical expressions for $\rho, T, R$ and $R_{\mathrm{bk}}$ can be obtained by transfer matrix or Fresnel coefficient methods ${ }^{14,16}$. 


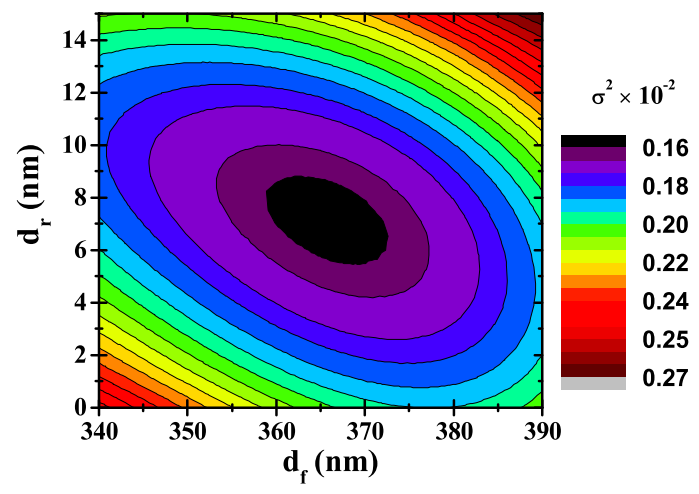

FIG. 2. Global unbiased error estimator $\sigma^{2}$ versus film and roughness layer thickness with a wavelength fitting range of $250 \mathrm{~nm}$ to $850 \mathrm{~nm}$ in steps of $5 \mathrm{~nm}$.

The procedure of minimizing $\sigma_{i}^{2}$ (Eq. 1) is repeated for different pairs of $d_{\mathrm{f}}$ and $d_{\mathrm{r}}$. In each case, the global unbiased error estimator $\sigma^{2}\left(d_{\mathrm{r}}, d_{\mathrm{f}}\right)$, defined in Eq. 4 , is calculated. The best fitted $d_{\mathrm{r}}$ and $d_{\mathrm{f}}$ are determined by the minimum $\sigma^{2}$.

$$
\sigma^{2}\left(d_{\mathrm{f}}, d_{\mathrm{r}}\right)=\frac{1}{9 \mathrm{~N}-3} \sum_{i=1}^{N} \sigma_{i}^{2}\left(n_{i}, k_{i}, d_{\mathrm{r}}, d_{\mathrm{f}}\right)
$$

Fig. 1 depicts the spectral measurements along with the theoretical curves after determining the best fit for $d_{\mathrm{r}}$ and $d_{\mathrm{f}}$. Note that $R_{\mathrm{bk}}$ differs from $R$ in the absorbing region, thus contributing as an additional constraint in this spectral region. $\sigma^{2}$ is plotted in Fig. 2, with a minimum for $d_{\mathrm{r}}=7.3 \pm 0.1 \mathrm{~nm}$ and $d_{\mathrm{f}}=365.3 \pm 0.1 \mathrm{~nm}$.

The latter estimation was performed in the wavelength range from $250 \mathrm{~nm}$ to $850 \mathrm{~nm}$, in which the nine spectra overlap. $\tilde{n}$ is determined then in three distinct regions due to the overlap of the spectrophotometry and VASE measurements. First, in the wavelength range from $190 \mathrm{~nm}$ to $250 \mathrm{~nm}$. In this case, $\tilde{n}$ was calculated using the VASE measurements only in the same previously described fashion. Second, in the wavelength range from $250 \mathrm{~nm}$ to $850 \mathrm{~nm}$, where $\tilde{n}$ was determined from all the acquired spectra. Third, from $850 \mathrm{~nm}$ to $2500 \mathrm{~nm}$ in which $\tilde{n}$ is determined, assuming $k=0$, from the $R, R_{\mathrm{bk}}$ and $T$ curves only. Since the material is transparent in this region and there was no overlap with additional measurements, we modeled $n$ with a Cauchy series up to the third order term. Fig. 3 depicts the resulting $n$ and $k$ calculated for the whole spectral region.

Fig. 4 and 5 compare $n$ and $k$ obtained in this work with those obtained by Shirayama et $\mathrm{al}^{1}$ and by Löper et $\mathrm{al}^{7}$. In the former, $\tilde{n}$ is obtained via spectral ellipsometry at $75^{\circ}$ 


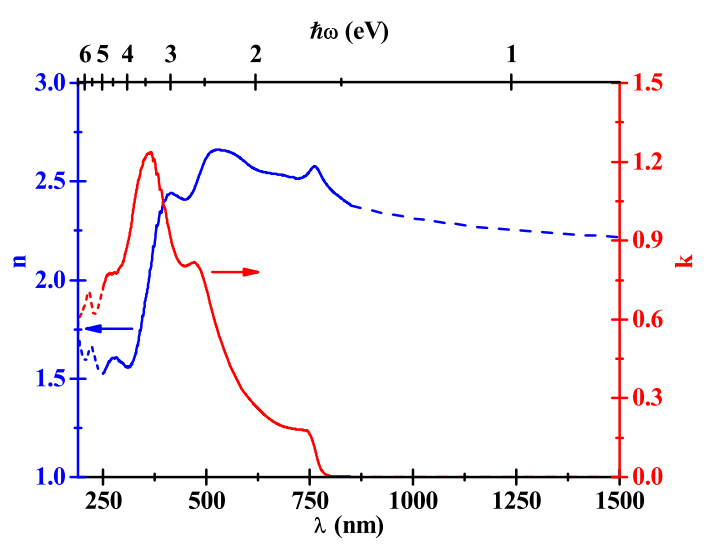

FIG. 3. Calculated $n$ and $k$. Solid lines correspond to the overlapping spectral region between $R$, $R_{\mathrm{bk}}, T$ and VASE measurements. Solid and dashed lines have the same meaning as in Fig. 1.

incident angle, in a nitrogen atmosphere, of two samples with different thicknesses, assuming the same optical constants. In the latter, $\tilde{n}$ is obtained via a global fit of $T, R$ and VASE using the Forouhi-Bloomer dispersion model. Additional $k$ data obtained by means of photo thermal deflection spectroscopy (PDS) from De Wolf et $\mathrm{al}^{13}$ is also included in Fig. 5 for comparison purposes. The shape and peak positions of our $\tilde{n}$ match with the previous results by Shirayama and Löper. The main differences between the obtained optical constants might be attributed to two features. First, in our case the measurements were carried out after exposing the samples to air for about one hour. Thus, as reported by Shirayama and others, some degradation has occurred. Second, the fit quality presented by Löper is partially limited, the fitted curves exhibit deviations at wavelengths around $330 \mathrm{~nm}, 550 \mathrm{~nm}$ and 900 nm. In Shirayama's work, $\epsilon_{2}$ had to be slightly adjusted by increasing the film thickness about $6 \%$ in order to have positive values below the bandgap. Furthermore, while the deposition method here and in Löper's publication are similar, in Shirayama's case the films were deposited by laser evaporation. Despite these differences, the extinction coefficients exhibit good correspondence with our results and of De Wolf, especially in the fundamental absorption region.

\section{BANDGAP DETERMINATION}

We determine the optical bandgap $E_{\mathrm{g}}$ and the Urbach energy $E_{\mathrm{u}}$ from the absorption coefficient calculated from our $\tilde{n}$ as $\alpha=4 \pi k / \lambda$, see Figs. 6 and 7. One of the main issues in 


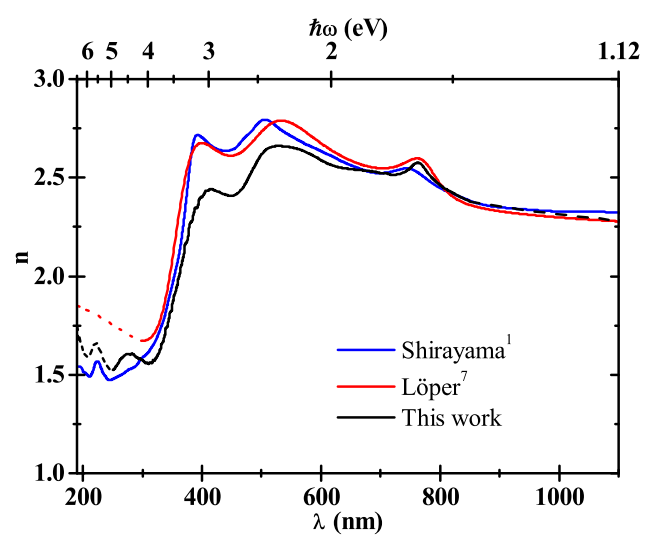

FIG. 4. Calculated refractive index. $n$ from Shirayama et $\mathrm{al}^{1}$ and Löper et $\mathrm{al}^{7}$ are also shown for comparison purposes. Dotted red line denotes an extrapolation below the minimum measured wavelength in the case of Löper. Dashed lines in black denote the regions where the VASE and $R$, $R_{\mathrm{bk}}$ and $T$ measurements don't overlap.

determining $E_{\mathrm{g}}$ from the fundamental absorption edge lies in the fact that an arbitrary linear region must be identified in the $(\alpha \hbar \omega)^{2}-$ plot $^{17}$. Although the latter method is simple and straightforward in comparison to a critical point analysis ${ }^{1}$, usually only few points remain in a rather small spectral region for fitting, as shown in the inset graph of Fig. 6. This is mainly due to the overlap of the Urbach tail with the band edge, thus making this method unreliable. However, it is still possible to extend the spectral region in consideration by including the Urbach tail in the fitting process. This can be achieved by using a band fluctuations average, in the free electron approximation, which serves to describe the shape of disorder induced localized states and extended states in a single equation ${ }^{18,19}$. Here we use our approach ${ }^{20,21}$, modified for crystalline semiconductors with direct bandgap. It consists of using an average JDOS to calculate the electronic transition rate and is described as follows.

Let $R_{\mathrm{cv}}$ and $D_{\mathrm{cv}}$ be the electronic transition rate and JDOS, respectively. At zero Kelvin and for direct transitions only, Fermi's golden rule is expressed by

$$
R_{\mathrm{cv}}=\mathcal{R} \sum_{k_{\mathrm{c}}, k_{\mathrm{v}}}\left|M_{\mathrm{cv}}\right|^{2} \delta\left(E_{c}-E_{v}-\hbar \omega\right) \delta_{k_{c}, k_{v}}
$$

or in integral form as

$$
R_{\mathrm{cv}}=\mathcal{R} \int\left|M_{\mathrm{cv}}\right|^{2} D_{\mathrm{cv}}\left(E_{\mathrm{cv}}\right) \delta\left(E_{\mathrm{cv}}-\hbar \omega\right) d E_{\mathrm{cv}}
$$




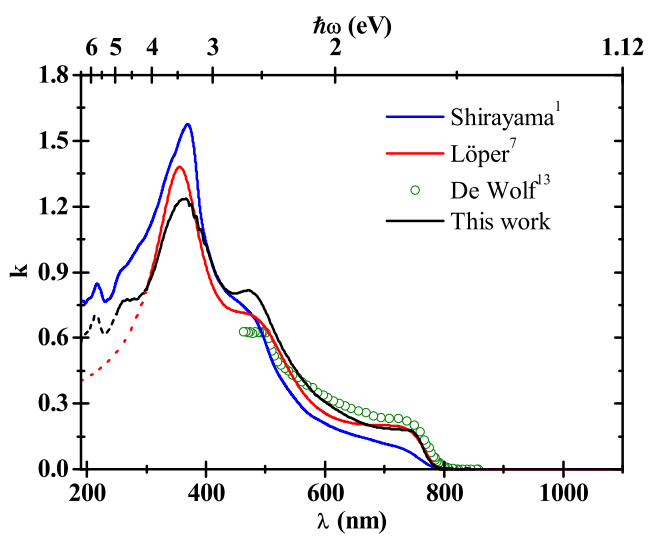

FIG. 5. Calculated $k$ in this work and from Shirayama et $\mathrm{al}^{1}$ and Löper et $\mathrm{al}^{7}$. Dotted red line denotes an extrapolation below the minimum measured wavelength in the case of Löper. Dashed line in black denote the region where $k$ was calculated from VASE only.

Here, $\mathcal{R}=2 \pi / \hbar\left(\overline{\mathrm{E}} e / 2 \omega m_{e}\right)^{2}$, with $m_{e}$ the electron mass, $e$ the elemental charge and $\overline{\mathrm{E}}$ the electric field of the incoming radiation. $E_{\mathrm{c}}$ and $E_{\mathrm{v}}$ are the conduction and valence energy bands in the free electron approximation, respectively. $\left|M_{\mathrm{cv}}\right|^{2}$ is the electronic transition matrix element. The definition of the joint energy band $E_{\mathrm{cv}}=E_{\mathrm{c}}-E_{\mathrm{v}}$ in this approximation allows to write the JDOS as shown in Eq. 7 , where $\mu^{*}$ is the reduced effective mass ${ }^{17}$.

$$
D_{\mathrm{cv}}\left(E_{\mathrm{cv}}\right)=\frac{\sqrt{2} \mu^{* 3 / 2}}{\pi^{2} \hbar^{3}} \begin{cases}\left(E_{\mathrm{cv}}-E_{\mathrm{g}}\right)^{1 / 2} & , E_{\mathrm{cv}}>E_{\mathrm{g}} \\ 0 & , E_{\mathrm{cv}} \leq E_{\mathrm{g}}\end{cases}
$$

We define the average electronic transition rate $\left\langle R_{c v}\right\rangle$ as shown in Eq. 8. Here $\left\langle D_{\text {cv }}\right\rangle$ is the average JDOS and is defined in Eq. 9 with $\widehat{W}$ the weight function accounting for the band fluctuations.

$$
\begin{gathered}
\left\langle R_{\mathrm{cv}}\right\rangle=\mathcal{R} \int\left|M_{\mathrm{cv}}\right|^{2}\left\langle D_{\mathrm{cv}}\right\rangle\left(E_{\mathrm{cv}}\right) \delta\left(E_{\mathrm{cv}}-\hbar \omega\right) d E_{\mathrm{cv}} \\
\left\langle D_{\mathrm{cv}}\right\rangle=\int D_{\mathrm{cv}}(\epsilon) \widehat{W}\left(\epsilon-E_{\mathrm{cv}}\right) d \epsilon
\end{gathered}
$$

A substitution of Eq. 9 in 8 leads to Eq. 10. Note that $\epsilon$ is a local (mute) integration variable and in this approximation we are assuming $\left|M_{\mathrm{cv}}\right|^{2}$ to be nearly constant versus the photon energy ${ }^{17}$. Therefore, Eq. 10 is equivalent to Eq. 6 with the exception of the weight 


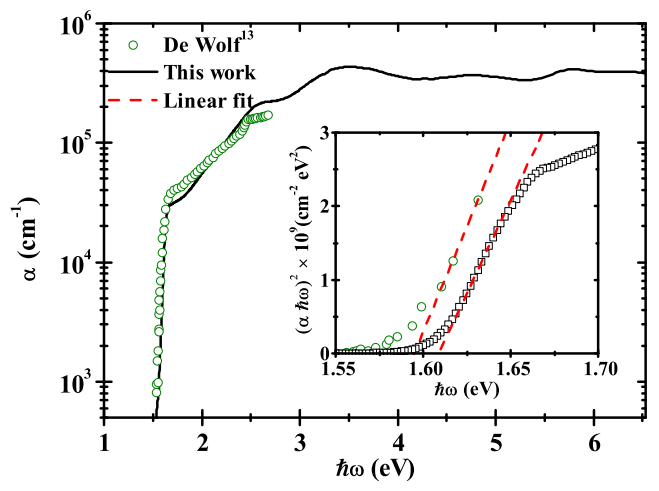

FIG. 6. Retrieved absorption coefficient and conventional direct absorption fit (inset graph). The PDS data of De Wolf ${ }^{13}$ is included for comparison purposes. Note the small spectral region with rather few data points to fit in the inset graph.

function. In the fluctuationless limit, the weight function must converge into a Dirac delta function $\widehat{W}\left(E_{\mathrm{cv}}-\hbar \omega\right) \rightarrow \delta\left(E_{\mathrm{cv}}-\hbar \omega\right)$ to fully recover Eq. 6 .

$$
\left\langle R_{c v}\right\rangle=\mathcal{R} \int\left|M_{\mathrm{cv}}\right|^{2} D_{\mathrm{cv}}(\epsilon) \widehat{W}(\epsilon-\hbar \omega) d \epsilon
$$

Here, instead of using a Gaussian distribution for describing thermal and/or structural band fluctuations ${ }^{18}$, we use our approach, in which we define $\widehat{W}(\epsilon)=-\partial f(\epsilon) / \partial \epsilon$, where $f(\epsilon)$ is the Fermi distribution function. Although this selection may seem arbitrary, it is inspired by the shape of the Kubo-Greenwood formula for the conductivity of amorphous materials ${ }^{22,23}$. While there is no direct relation between these equations, in the latter, the effect of the occupation degree of an ensemble of electrons at finite temperatures on the electronic transition rate leads to an expression similar to that of Eq. 10.

$\widehat{W}(\epsilon)$ not only exhibits a Gaussian like behaviour and becomes $\delta(\epsilon)$ in the fluctuationless limit, but actually enables to write Eq. 10 in terms of the Fermi-Dirac integral $\left(\mathrm{F}_{j}\right)$ which has the advantage of being feasible to implement in a least-square fitting procedure. $\mathrm{F}_{j}$ is defined in Eq. 11 and the result for the absorption coefficient is depicted in Eq. 12 after $\alpha(\hbar \omega) \propto R_{\mathrm{cv}}(\hbar \omega) \hbar \omega$.

$$
\begin{aligned}
\mathrm{F}_{j}(x) & =\frac{1}{\Gamma(j+1)} \int_{0}^{\infty} \frac{t^{j}}{\exp (t-x)+1} d t \\
& =-\mathrm{Li}_{j+1}(-\exp (x))
\end{aligned}
$$




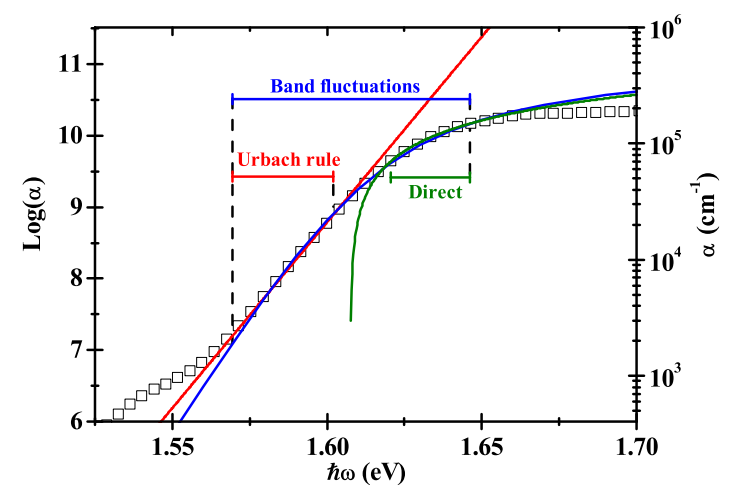

FIG. 7. Fitted absorption coefficient using the traditional Urbach rule (red), band fluctuations model (blue) and conventional direct absorption model (green).

$$
\alpha(\hbar \omega)=-\frac{1}{2} \frac{\alpha_{0}}{\hbar \omega} \sqrt{\frac{\pi}{\beta}} \operatorname{Li}_{1 / 2}\left(-\mathrm{e}^{\beta\left(\hbar \omega-E_{\mathrm{g}}\right)}\right)
$$

Here, $\Gamma(x)$ is the gamma function of $x, \operatorname{Li}_{j}(x)$ is the $j$-th order polylogarithm function of $x$, the coefficients $\mathcal{R}$, those from $D_{\mathrm{cv}}$, and $\left|M_{\mathrm{cv}}\right|^{2}$ are absorbed in the coefficient $\alpha_{0}$. $\beta$ is the Urbach slope and is inherited from the Fermi distribution. It defines how large the fluctuations are.

Note that the model contains only three fitting parameters, $\alpha_{0}, E_{\mathrm{g}}$ and $\beta$. These can be determined with a single fit of the fundamental absorption. Furthermore, an asymptotic analysis of Eq. 12 leads to the conventional direct absorption coefficient for band-to-band transitions and to an exponential tail in the bandgap (see Eq. 13).

$$
\alpha(\hbar \omega) \approx \frac{\alpha_{0}}{\hbar \omega} \begin{cases}\frac{1}{2} \sqrt{\frac{\pi}{\beta}} e^{\beta\left(\hbar \omega-E_{\mathrm{g}}\right)} & , \hbar \omega \ll E_{\mathrm{g}} \\ \left(\hbar \omega-E_{\mathrm{g}}\right)^{1 / 2} & , \hbar \omega \gg E_{\mathrm{g}}\end{cases}
$$

Fig. 7 depicts fits using the conventional $(\alpha \hbar \omega)^{2}$-plot method, the Urbach rule, $\alpha \propto$ $\exp (\beta \hbar \omega)$, and Eq. 12 on our retrieved absorption coefficient. Note the distinct spectral regions involved. The fits of Eq. 12 on the absorption coefficient obtained by Shirayama, Löper and De Wolf are presented in Fig. 8. Table I summarizes the retrieved $E_{\mathrm{g}}$ and $E_{\mathrm{u}}$. Note that fits with the conventional model predict an identical bandgap from the absorption data given by De Wolf, Löper and this work. These values are very susceptible to the selected fitting region ${ }^{1}$. On the other hand, the fits with Eq. 12 reveal bandgap values that follow the trend observed in Fig. 8. Additionally, the Urbach slope is equally susceptible to the 


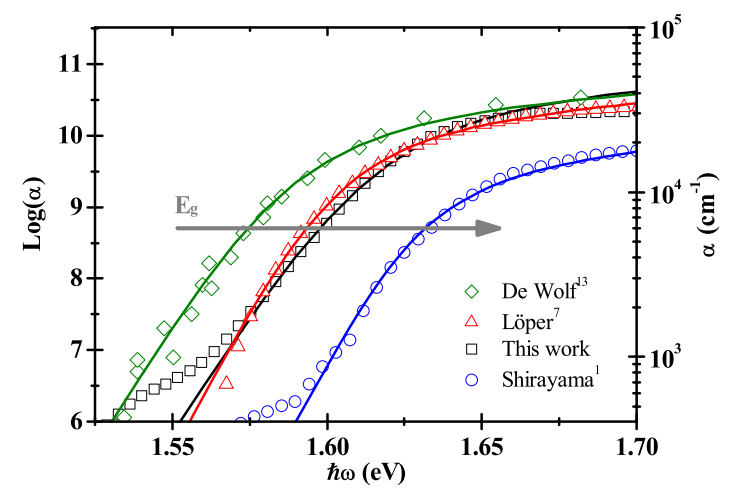

FIG. 8. Fitted absorption coefficients using the band-fluctuations model (Eq. 12). The associated $E_{\mathrm{g}}$ and $E_{\mu}$ are sumarized in Table I. The arrow denotes the bandgap increase.

TABLE I. $E_{\mathrm{g}}$ and $E_{\mathrm{u}}$ values after fitting the absorption coefficient depicted in Fig. 8. Upper labels $\mathrm{CO}$, UR and BF stand for conventional, Urbach rule and band fluctuations, respectively. Errors associated to ${ }^{\mathrm{BF}} E_{\mathrm{g}}$ are around $0.001 \mathrm{eV}$.

\begin{tabular}{ccccc}
\hline \hline Source & ${ }^{\mathrm{CO}} E_{\mathrm{g}}(\mathrm{eV})$ & ${ }^{\mathrm{UR}} E_{\mathrm{u}}(\mathrm{meV})$ & ${ }^{\mathrm{BF}} E_{\mathrm{g}}(\mathrm{eV})$ & ${ }^{\mathrm{BF}} E_{\mathrm{u}}(\mathrm{meV})$ \\
\hline Wolf $^{13}$ & $1.60 \pm 0.11$ & $14.8 \pm 0.3$ & 1.579 & $14.3 \pm 0.2$ \\
Löp. $^{7}$ & $1.60 \pm 0.01$ & $10.7 \pm 0.3$ & 1.595 & $12.0 \pm 0.2$ \\
TW & $1.61 \pm 0.01$ & $19.3 \pm 0.1$ & 1.607 & $14.9 \pm 0.3$ \\
Shir. $^{1}$ & $1.63 \pm 0.07$ & $14.4 \pm 1.0$ & 1.623 & $12.4 \pm 0.3$ \\
\hline \hline
\end{tabular}

fitting region. These issues are overcome when using the band fluctuations model. We believe this model will be useful for the experimentalist and it could be extended to consider a Kramers-Kronig consistent calculation for the refractive index ${ }^{24}$.

\section{CONCLUSIONS}

The results presented in this work can be summarized in two parts. First, we have shown that it is possible to determine $\tilde{n}(\lambda)$ with high accuracy and without using a dispersion model from a single sample, by performing a minimization of the unbiased global error estimator defined from $T, R$ and $R_{\mathrm{bk}}$ spectra along with VASE spectra. The retrieved complex refractive index was then compared to those recently reported by Shirayama ${ }^{1}$ and 
Löper ${ }^{7}$. In the former case, the samples were grown by laser evaporation, and $\tilde{n}(\lambda)$ was determined by a self consistent analysis of spectral ellipsometry of two samples with the same optical constants but different thicknesses. On the other hand, in Löper's case, the sample was grown by a process similar to the one used in this work, and $\tilde{n}(\lambda)$ was determined after fitting $R$ and $T$ and VASE spectra with the Forouhi-Bloomer dispersion model. The differences were exposed previously and are attributed to both the deposition process and the different calculation methods for optical constants.

Second, we have developed a straightforward method for determining the optical bandgap and Urbach energy from the fundamental absorption. The model has only three fitting parameters (see Eq. 12) and keeps the simplicity of the conventional $(\alpha \hbar \omega)^{2}$-plot method making it easy to implement in a least-square fitting procedure. Fits of the absorption coefficient obtained in this work and of those by Löper ${ }^{7}$, Shirayama ${ }^{1}$ and De Wolf ${ }^{13}$ show a good correspondence between model and experiment (see Fig. 8). The bandgap and Urbach energy retrieved by means of the latter model are summarized in Table I.

\section{ACKNOWLEDGMENTS}

This work was funded by the German Academic Exchange Service (DAAD), and the Peruvian National Fund for Scientific and Technological Development (FONDECYT), grant no. 037-2016. Additional support has been provided by the Research Internationalization Office of the Pontificia Universidad Católica del Perú, as well as the German Federal Ministry of Education and Research (BMBF), within the project "Materialforschung für die Energiewende", grant no. 03SF0540. The authors also acknowledge the Peruvian National Program for Innovation, Competitiveness and Production (PNCIP), contract no. 274-PNICP-BRI-2015.

\section{REFERENCES}

${ }^{1}$ M. Shirayma et al, Phys. Rev. Applied 5(1), 014012 (2016).

${ }^{2}$ M. A. Green et al, Nature Photon. 8(7), 506-514 (2014).

${ }^{3}$ Q. Dong et al, Science 347(6225), 967-970 (2015).

${ }^{4}$ J. Burschka et al, Nature 499(7458), 316-319 (2013). 
${ }^{5}$ W. E. I. Sha et al, Appl. Phys. Lett. 106(22), 21104 (2015).

${ }^{6}$ R. Asadpour et al, Appl. Phys. Lett. 106(24), 243902 (2015).

${ }^{7}$ P. Loper et al, J. Phys. Chem. Lett. 6(1), 66-71 (2015).

${ }^{8}$ P. Loper et al, IEEE J. Photovolt. 4(6), 1545-1551 (2014).

${ }^{9}$ S. Albrecht et al, Energy Environ. Sci. 9(1), 81-88 (2015).

${ }^{10}$ J. H. Heo et al, Adv. Mater. 26(46), 8179-8183 (2014).

${ }^{11}$ G. E. Eperon et al, Energy Environ. Sci. 7(3), 982-988 (2014).

${ }^{12}$ N. J. Jeon et al, Nat. Mater. 13(9), 897-903 (2014).

${ }^{13}$ S. De Wolf et al, J. Phys. Chem. Lett 5(6), 1035-1039 (2014).

${ }^{14}$ H. Fujiwara, in Spectroscopic Ellipsometry: Principles and Applications (Wiley, West Sussex, U.K., 2007).

${ }^{15}$ R. M. Dawson et al, Mat. Res. Soc. Symp. Proc. 258 595-600 (1992).

${ }^{16}$ R. Vitlina and G. Surdutovich, Appl. Opt. 41(16) 3111-3117 (2002).

${ }^{17} \mathrm{P}$. Yu and M. Cardona, in Fundamentals of Semiconductors: Physics and Materials Properties Third, Revised and Enlarged Edition (Springer, Berlin, 2005).

${ }^{18}$ S. K. O’Leary et al, Phys. Rev. B 52(11), 7795-7797 (1995).

${ }^{19}$ J. J. Thevaril et al, J. Appl. Phys. 120(13), 135706 (2016).

${ }^{20}$ J. A. Guerra et al, J. Phys. D: Appl. Phys 49(19), 195102 (2016).

${ }^{21}$ J. A. Guerra et al, MRS Adv. 1(43), 2929-2934 (2016).

${ }^{22}$ P. B. Allen, in Conceptual Foundations of Materials: A Standard Model for Ground- and Excited-State Properties, Chapter 6, (Elsevier, B.V., 2006).

${ }^{23}$ L. L. Moseley et al, Am. J. Phys. 46(6), 676-677 (1978).

${ }^{24}$ A. S. Ferlauto et al, J. App. Phys. 92(5), 2424-2436 (2002). 